\title{
ANÁLISIS Y TRATAMIENTO DE SEÑALES DE FUENTES SISMOGÉNICAS DE CAMPO CERCANO A SAN JOSÉ DE CÚCUTA, COLOMBIA
}

Por: C. H. Flórez Góngora ${ }^{1}$, C. F. Lozano Lozano².

\section{RESUMEN}

Esta investigación determina el contenido frecuencial dominante de un conjunto de 69 señales de fuentes sismogénicas cercanas a la ciudad de San José de Cúcuta, obtenidas mediante la Red Nacional de Acelerógrafos de Colombia (RNAC) perteneciente al Instituto Colombiano de Geología y Minería (INGEOMINAS). Se realizó análisis y tratamiento para cada una de las señales. La etapa de tratamiento comprende los procesos de corrección por línea base y el filtrado de la señal sísmica para eliminar bandas de frecuencias no deseadas presentes en la señal. Las técnicas utilizadas para el análisis espectral son la transformada de Fourier y la transformada Wavelet, mediante sus respectivos espectros de amplitudes. $Y$ se simula el efecto local de los sismos analizados para dos perfiles de suelo de formaciones litológicas diferenciadas. Los análisis se realizaron bajo el uso de herramientas computacionales; programas como EERA, SeismoSignal, DEGTRA A4 y MATLAB ${ }^{\circledR}$.

Palabras Claves: Señal sísmica, transformada de Fourier, transformada wavelet, espectro de respuesta.

\section{ABSTRACT}

This research determines the dominant frequency content of a set of 69 signals from seismogenic sources close to the city of San José de Cúcuta, the signals were gathered by the Colombian National Network of Acelerograph (RNAC in Spanish) belonging to the Colombian Institute of Geology and Mining (INGEOMINAS in Spanish). Treatment and analysis was conducted for each of the signals. The stage of treatment involves processes for baseline correction and filtering of the seismic signal to remove unwanted frequency bands present in the signal. The techniques used for spectral analysis are the Fourier transform and Wavelet transform, through their respective amplitude spectra. It simulates the effect of local earthquakes analyzed for two soil profiles of different lithological formations. Analysis was performed under the use of computational tools; programs like EERA, SeismoSignal, DEGTRA A4 and MATLAB ${ }^{\circledR}$.

Key Words: Seismic signal, Fourier transform, wavelet transform, response spectrum.

\section{INTRODUCCIÓN}

El presente proyecto de investigación, analiza el fenómeno sísmico que, consiste en el proceso de liberación de energía acumulada lentamente en los bordes de las placas tectónicas que conforman la superficie terrestre producto de sus desplazamientos, o también, esta energía puede ser liberada por una serie de fallas geológicas internas [1] [2] [3] [4], donde se ubican las denominadas fuentes sismogénicas.

La señal sísmica o acelerograma, es el resultado del mecanismo de ruptura en la fuente sismogénica, contiene parámetros sísmicos de importancia tales como el contenido frecuencial y la aceleración en el sitio donde se construyen nuestras estructuras, además, esta señal es modificada por los distintos medios que atraviesa, hasta poder establecer una relación en sus frecuencias dominantes.

El campo cercano, se define como la región en torno a la fuente sísmica situada a una distancia más pequeña que la longitud de la fractura, parámetro que se relaciona directamente con la magnitud del

\footnotetext{
${ }^{1}$ Ingeniero Civil, M.Sc. Profesor Investigador. Grupo de Investigación en Geotecnia Ambiental - GIGA, Departamento de Geotecnia y Minería, Universidad Francisco de Paula Santander. chflorez@bari.ufps.edu.co.

${ }^{2}$ Estudiante Ingeniería Civil. Grupo de Investigación en Geotecnia Ambiental - GIGA, Universidad Francisco de Paula Santander. carloslozano53@yahoo.es.
} 


\title{
ANÁLISIS Y TRATAMIENTO DE SEÑALES DE FUENTES SISMOGÉNICAS DE CAMPO CERCANO A SAN JOSÉ DE
}

\author{
CÚCUTA, COLOMBIA
}

sismo [5]. Las ondas sísmicas poseen una elevada frecuencia y por tanto una pequeña longitud de onda (periodos cortos) [6].

La ciudad de San José de Cúcuta, capital del departamento de Norte de Santander, está ubicada en una zona de alta sismicidad con una aceleración pico efectiva horizontal de diseño de $0.30 \mathrm{~g}$ [7], además, puede verse sometida a un sismo producto de la convergencia de las fallas geológicas de Boconó (Venezuela) y la Falla Frontal de la Cordillera Oriental (Colombia) [8]. La Norma Colombiana de Construcciones Sismorresistentes (NSR - 98) exige llevar a cabo estudios de rigor sobre los distintos aspectos técnicos y científicos que cubre un estudio completo de Microzonificación Sísmica. Por lo tanto, esta investigación estudia las fuentes sismogénicas cercanas a nivel frecuencial para la determinación de patrones que pueden aportar información importante sobre la naturaleza del tren ondulatorio.

Tomando como referencia la ciudad de San José de Cúcuta, se realiza una modelación teórica de la propagación de ondas sísmicas a través del suelo; empleando señales registradas en la ciudad y otro conjunto de señales correspondientes a sismos fuertes registrados en diversas partes del mundo, estableciendo la respuesta de los suelos de Cúcuta ante la eventual ocurrencia de un movimiento telúrico de dimensiones catastróficas.

\section{MATERIALES Y MÉTODOS}

Los archivos correspondientes a los registros sísmicos tienen extensión.evt, para lo cual se emplea el programa Kw2cnts de Kinemetrics para extraer los datos de la señal sísmica y convertirlos a un archivo de texto. Se realizan operaciones como la corrección por línea base y se pueden cambiar las unidades de aceleración [9]. Para verificar el correcto funcionamiento del programa Kw2cnts, se hacen comparaciones con la versión demo del programa
SMA (Strong Motion Analyst) de Kinemetrics, arrojando excelentes resultados.

Ejecutadas las operaciones anteriormente descritas, para finalizar el proceso de tratamiento de la señal, se aplica un filtro para eliminar las frecuencias que están fuera del rango de medición del aparato. El filtrado elimina la información errada de baja frecuencia que se genera en los aparatos de registro, el ruido de baja y alta frecuencia de los sitios de registro, y las amplitudes de alta frecuencia que no son de interés para el análisis de la respuesta dinámica de estructuras y depósitos de suelo [10] [11] [12] [13].

Una vez realizadas las correcciones y el tratamiento de la señal, se obtiene el acelerograma base corregido para cada una de las componentes y con base en ellos se utilizan programas de computador y procedimientos numéricos para obtener el espectro de amplitudes de Fourier y el espectro de la transformada wavelet. En el anexo 1 se muestra un conjunto de señales sísmicas procesadas que se registraron en la ciudad de San José de Cúcuta.

\subsection{Parámetros utilizados para el análisis y tratamiento de la señal sísmica}

\subsubsection{Procesamiento de la señal sísmica}

La extracción de la información, la conversión a archivos en formato ASCll y la corrección por línea base, se hizo con el programa kw2cnts de Kinemetrics; para esta investigación, se extrajeron los 3 canales de registro (Este - Oeste, Vertical, Norte- Sur), pero tan solo se procesaron dos componentes, Este - Oeste y Vertical; luego de realizar este procedimiento, se convierten las unidades de Gales $\left(\mathrm{cm} / \mathrm{s}^{2}\right)$ a unidades de gravedad $\left(\mathrm{g}=1000 \mathrm{~cm} / \mathrm{s}^{2}\right)$. Para el proceso de filtrado de la señal sísmica, se emplea el programa SeismoSignal [14], escogiendo un filtro de tipo Butterworth, de orden 6 y con un rango de frecuencias de corte de 0.25 a $50 \mathrm{~Hz}$, el cual coincide con el rango de respuesta del acelerógrafo $(0-50 \mathrm{~Hz})$. 


\section{ANÁLISIS Y TRATAMIENTO DE SEÑALES DE FUENTES SISMOGÉNICAS DE CAMPO CERCANO A SAN JOSÉ DE}

\section{CÚCUTA, COLOMBIA}

\subsubsection{Modelo para respuesta local}

Para determinar la respuesta de la señal sísmica en la superficie y de esta manera establecer los niveles de amplificación de los suelos de la ciudad de San José de Cúcuta, debido al paso de las ondas sísmicas a través del suelo, y como la mayoría de estas señales son registradas en roca, se realiza el análisis unidimensional de propagación de onda. La información utilizada para el análisis de los perfiles litológicos, se extrajo de perforaciones, estudios e investigaciones y trabajos de grado que han recopilado información sobre caracterización geotécnica de los suelos de la ciudad de Cúcuta [15] [16] [17], a los cuales se les calcularon los parámetros dinámicos de forma teórica.

\subsubsection{Información geotécnica}

La localización geográfica de los perfiles empleados para el

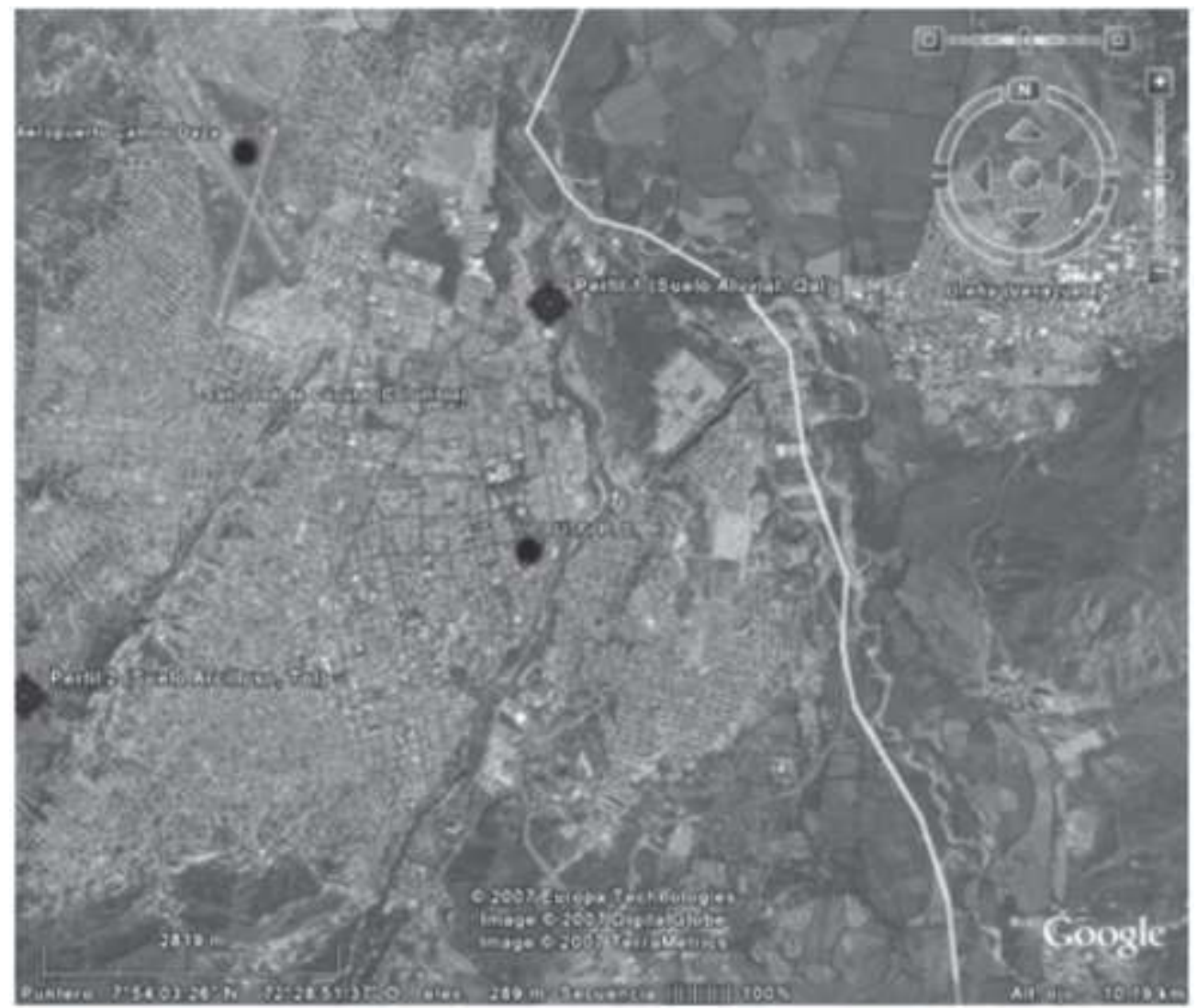

análisis de la propagación

unidimensional, se ilustra en la figura 1 . Se utilizaron dos perfiles geotécnicos para la modelación de la propagación unidimensional de onda. El perfil 1 corresponde a terrazas del cuaternario reciente (Qal), constituidas por material aluvial depositados por corrientes principales superficiales en diferentes niveles topográficos, siendo la principal de ellas, el río Pamplonita. [18]. El perfil 2 pertenece a la formación León, que se constituye por un conjunto de arcillolitas abigarradas, microlaminadas de media

a alta plasticidad, con láminas delgadas de yeso y trazas carbonáceas, ínter estratificadas; las arcillas de estos grupos, son sedimentos altamente erosionables y susceptibles a experimentar cambios volumétricos por modificaciones en su contenido de humedad [18].

Figura 1. Localización geográfica de los perfiles analizados.

Fuente: Google Earth (2007). Localización: Autores.

\subsubsection{Parámetros dinámicos}

Los dos perfiles de suelo se someten al análisis de propagación unidimensional de onda; se calculan teóricamente sus propiedades dinámicas, utilizando los modelos hiperbólicos de suelos de Hardin y Drnevich (1972) para las arcillas, Hardin y Black (1968) para las arenas, y Seed et al. (1972) para las gravas [19]. 


\section{ANÁLISIS Y TRATAMIENTO DE SEÑALES DE FUENTES SISMOGÉNICAS DE CAMPO CERCANO A SAN JOSÉ DE}

\section{CÚCUTA, COLOMBIA}

Figura 2. Composición estratigráfica para el perfil de suelo aluvial (Perfil 1).
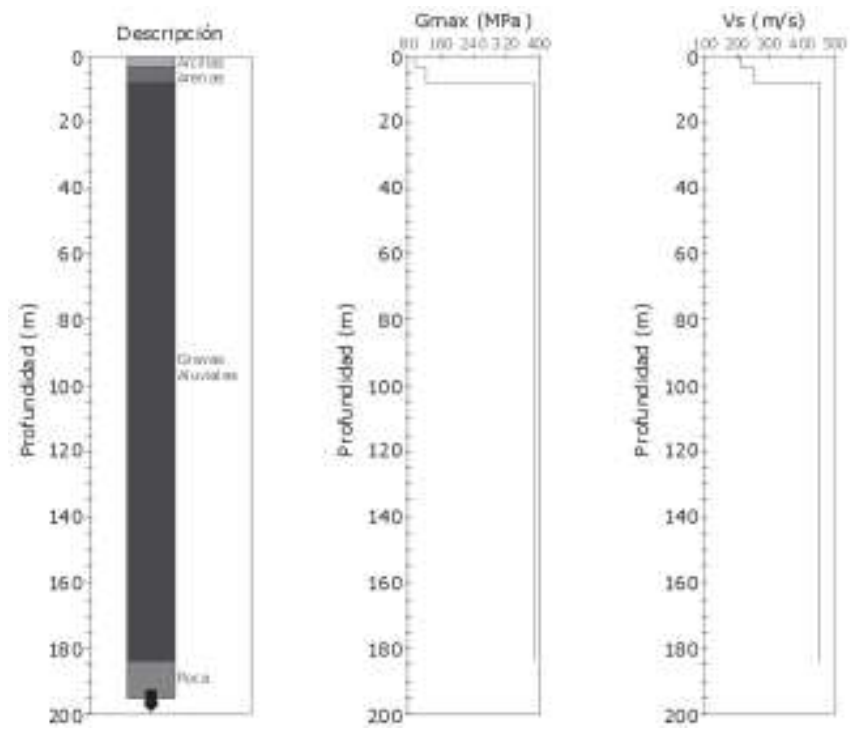

Fuente: Autores.

Figura 3. Composición estratigráfica para el perfil de suelo arcilloso (Perfil 2).
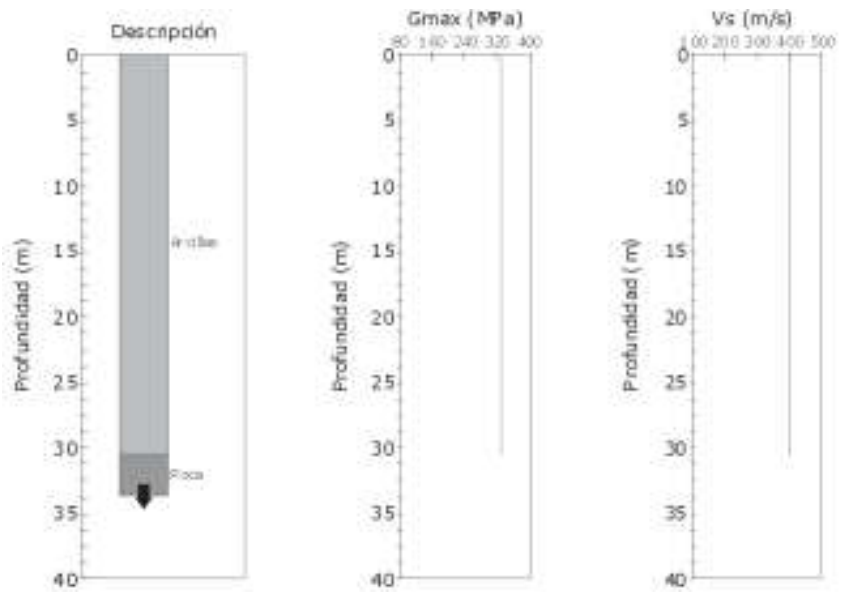

Fuente: Autores.

Las figuras 2 y 3, muestran la composición de los perfiles utilizados para el análisis, la profundidad de cada uno de los estratos, la variación del módulo cortante máximo y la velocidad de onda cortante respecto a la profundidad, para el perfil de suelo aluvial (perfil 1) y para el perfil de suelo arcilloso (perfil 2), respectivamente.

\subsubsection{Análisis Frecuencial}

El programa EERA [20], realiza la modelación de la propagación unidimensional de onda, calcula el espectro de Fourier y el espectro de respuesta. Se emplean los programas SeismoSignal [14], DEGTRA A4 [21], NONLIN [22] y EDUSHAKE [23] para realizar análisis comparativos en lo que tiene que ver con el cálculo de frecuencias dominantes y los espectros de respuesta, con los cuales se obtuvieron resultados similares, confirmando que los procesos realizados se hicieron de forma correcta. Mediante un algoritmo sencillo en el programa Matlab ${ }^{\circledR}$, se realiza el cálculo de la transformada wavelet continua (CWT), que corresponde al tipo de transformada que se utilizó para el análisis de la señal sísmica objeto de estudio para esta investigación.

\subsubsection{Análisis frecuencial mediante la} transformada rápida de Fourier (FFT)

La ecuación (1) es conocida como la transformada de Fourier (también llamada como transformada de Fourier compleja o espectro de Fourier) y la ecuación (2) es la transformada inversa de Fourier [10] [12] [24] [25] [26] [27].

$$
\begin{gathered}
F(\omega)=\int_{-\infty}^{\infty} f(t) e^{-i \omega t} d t \\
f(t)=\frac{1}{2 \pi} \int_{-\infty}^{\infty} F(\omega) e^{i \omega t} d \omega
\end{gathered}
$$

Considerando una secuencia $x(n)$ [10] [12] [24] [27]. Su transformada discreta de Fourier (DFT) de N-puntos es dada por:

$$
x(k)=\sum_{n=0}^{N-1} x(n)^{-r(2 \pi / N) n k}, k=0,1, \ldots, N-1
$$

El análisis mediante la transformada rápida de Fourier (FFT), se realizó con el programa EERA, este utiliza el algoritmo propuesto por Cooley y Tukey [20] [28]. 


\section{ANÁLISIS Y TRATAMIENTO DE SEÑALES DE FUENTES SISMOGÉNICAS DE CAMPO CERCANO A SAN JOSÉ DE} CÚCUTA, COLOMBIA

El parámetro que se tiene en cuenta es la definición del número de puntos $\mathrm{N}$ para el cálculo de la FFT, ver la ecuación (3). Generalmente el valor de N debe ser mayor que el número de puntos de la señal sísmica a analizar, en este caso, la señal de entrada es agregada con ceros para producir una señal de longitud N. es recomendable para un cálculo rápido, escoger $\mathrm{N}$ como una potencia de dos.

\subsubsection{Análisis frecuencial mediante la transformada Wavelet (WT)}

Una wavelet es una función de promedio cero:

$$
\int_{\infty}^{+\infty} \psi(t) d t=0
$$

la función de transformación : $\psi_{i s}(t)$

$$
\psi_{u, s}(t)=\frac{1}{\sqrt{s}} \psi\left(\frac{t-u}{s}\right)
$$

La transformada wavelet continua se define matemáticamente como:

$$
C(u, s)=\int_{-\infty}^{+\infty} f(t) \frac{1}{\sqrt{s}} \psi\left(\frac{t-u}{s}\right) d t
$$

De la ecuación (5) se observa la función de transformación denominada wavelet madre, siendo $\mathrm{s}$ un parámetro de escala y u de traslación. La ecuación (6) muestra la transformada wavelet continua (CWT). Los resultados de la CWT son los coeficientes $C(u, s)$ de $f(t)$ en el espacio de funciones definido por $v_{i,}(t)$ [29] [30] [31] [32] [33]. Para el cálculo de la transformada wavelet continua (CWT), se emplea un algoritmo en el programa MATLAB ${ }^{\circledR}$; se escoge una wavelet madre, en este proyecto se utiliza la wavelet de Daubechies de orden 6 (db6), la cual se asemeja en su forma a una señal sísmica; además se escoge un valor de escala de 64 para el análisis de las señales.

\subsubsection{Cálculo de espectros derespuesta}

La forma más completa de representación del movimiento para fines de diseño es por medio de espectros de respuesta, que indican la respuesta máxima de osciladores simples de un grado de libertad con cierto amortiguamiento, ante una excitación sísmica, en función de la frecuencia propia del oscilador. Dicha respuesta puede expresarse en términos de aceleración, velocidad o desplazamiento para las distintas frecuencias del movimiento [4] [34] [35].

\section{Resultados}

Para efectos de ilustración se presentan los diversos resultados para la componente Este - Oeste (EW), aunque el análisis completo se hizo para las componentes Este-Oeste (EW) y Vertical (V) de señales registradas en roca y en superficie, y para las señales analizadas mediante la modelación unidimensional de propagación de onda.

\subsection{Espectro de amplitudes de Fourier}

Mediante los espectros de amplitudes de Fourier es posible conocer las frecuencias dominantes de la señal, que corresponde al valor de la máxima amplitud, determinando su correspondiente frecuencia. Se calculan las frecuencias dominantes para las señales sísmicas a nivel de roca y suelo, según el sitio de registro, como también para los dos perfiles analizados para la ciudad de San José de Cúcuta.

\subsubsection{Contenido Frecuencial Dominante}

Para clasificar los sismos registrados en la ciudad, se decidió hacer la siguiente distribución según la fuente sísmica y la distancia epicentral. Como el $70 \%$ de los sismos registrados pertenecen al Nido de Bucaramanga, se calculan las frecuencias dominantes 


\title{
ANÁLISIS Y TRATAMIENTO DE SEÑALES DE FUENTES SISMOGÉNICAS DE CAMPO CERCANO A SAN JOSÉ DE
}

\author{
CÚCUTA, COLOMBIA
}

para esta fuente sismogénica; para sismos con distancias epicentrales menores a $200 \mathrm{Km}$, que corresponden a sismos locales o cercanos y sismos con distancias epicentrales mayores a $200 \mathrm{Km}$.

En el anexo 2, se muestra la distribución de frecuencias dominantes, relacionada con la profundidad $(\mathrm{Km})$ y la distancia epicentral $(\mathrm{Km})$, correspondientes a los sismos registrados en las estaciones CCUC1 y CCUC3, de los cuales se tomaron registros sísmicos a nivel de roca. En la tabla 1, se presentan las características de las estaciones ubicadas en la ciudad de Cúcuta, entre los cuales encontramos, el tipo de instrumento (acelerógrafo), la geología y la topografía del sitio sobre el cual se encuentran emplazadas las estaciones.

Tabla 1. Características generales de las estaciones ubicadas en la ciudad de Cúcuta. procesamiento. Para las señales registradas en roca se tuvo en cuenta la respuesta de la señal en superficie, es decir, se determinó el espectro de la transformada wavelet para las señales modeladas mediante la propagación unidimensional de onda, para el perfil de suelo aluvial (perfil 1) y el perfil de suelo arcilloso (perfil 2). En el anexo 4, se muestran los espectros de la transformada wavelet, el cual se representa en el plano Tiempo - Escala, lo cual nos permite determinar en que instantes de tiempo aparecen las frecuencias dominantes de la señal; la duración en el tiempo se obtiene multiplicando el valor que aparece en el eje horizontal por el intervalo de registro que corresponde a 0.005 segundos

\subsection{Espectros de Respuesta}

El anexo 5, muestra los espectros de respuesta calculados para el $5 \%$ de amortiguamiento con

\begin{tabular}{|c|c|c|c|c|c|c|c|}
\hline Nombre de la Estación & Código & Geologia & LAT $\left(^{\circ}\right)$ & LONG $\left(^{\circ}\right)$ & Topografia & $\begin{array}{c}\text { Tipo de } \\
\text { Instrumento }\end{array}$ & Propietario \\
\hline Cúcuta (Circunvalación) & CCUC1 & Roca & 7.88 & -72.51 & Ondulada & ETNA & INGEOMINAS \\
\hline Cúcuta (UFPS) & CCUC2 & Suelo & 7.90 & -72.49 & Plana & SSA - 2MC & INGEOMINAS \\
\hline Cúcuta (Santo Domingo) & CCUC3 & Roca & 7.874 & -72.508 & Ondulada & ETNA & INGEOMINAS \\
\hline
\end{tabular}

Fuente: Red Nacional de Acelerógrafos de Colombia, 2006.

\subsubsection{Espectros de Fourier para los perfiles de suelo analizados}

Se obtuvieron gráficos de los espectros de Fourier, los cuales se muestran en el anexo 3, en estas figuras se observa que el espectro corresponde a un plano frecuencia - amplitud, el eje de las abscisas representa la Frecuencia $(\mathrm{Hz})$ y el eje de las ordenadas la Amplitud de Fourier.

\subsection{Espectro de la transformada wavelet}

El análisis espectral con la transformada wavelet, se realizó para las cinco señales con mayor valor de aceleración en unidades de gravedad, ya que este es un proceso que requiere de mucho tiempo para el respecto al crítico, se analiza la respuesta para las componentes EW y Vertical del sismo, y para los dos perfiles correspondientes al suelo aluvial y al suelo arcilloso. Se observa que en el plano donde se ubica el espectro, el eje de las abscisas representa el periodo (segundos) y el eje de las ordenadas la aceleración espectral en unidades de la gravedad.

\subsection{Espectros de respuesta para registros de movimiento fuerte del suelo}

Para conocer el verdadero potencial de amplificación de los suelos de la ciudad, se realiza una modelación de la propagación unidimensional de onda, para ocho registros de movimiento fuerte del suelo a nivel mundial, que corresponden a señales sísmicas de terremotos históricos, los cuales son de alta magnitud y aceleración. Los espectros se muestran en el anexo 6 y estos movimientos corresponden a los sismos de: 
ANÁLISIS Y TRATAMIENTO DE SEÑALES DE FUENTES SISMOGÉNICAS DE CAMPO CERCANO A SAN JOSÉ DE CÚCUTA, COLOMBIA

Imperial Valley - El Centro (USA); Ciudad de México (México); Loma Prieta (USA); Northridge (USA); San Fernando - Pacoima Dam (USA); San Fernando Orion BLVD (USA); Valparaíso (Chile); Kobe (Japón).

\section{Análisis deResultados}

4.1. Resultados para el análisis espectral de fourier

Comparando los valores de frecuencias dominantes en roca y para los perfiles de suelo analizados, de las tablas 2 y 3 , respectivamente, determinamos que la señal sísmica al propagarse por el perfil de suelo 1 , conserva sus componentes frecuenciales hasta llegar a la superficie; en cambio para el perfil 2 se observa que este modifica el contenido frecuencial de la señal registrada en roca, es decir, conserva solo unas componentes de frecuencia, como es el caso del rango entre 3.0 y $4.0 \mathrm{~Hz}$ y desecha las demás.
4.2. Resultados para el análisis espectral mediante la transformada wavelet

Para este análisis se escogieron tres señales captadas en roca y dos captadas en suelo, es decir, en superficie. También se le realizó el análisis de la transformada wavelet, a las señales producto del análisis de propagación unidimensional de onda, correspondientes a los perfiles de suelo aluvial (perfil 1) y arcilloso (perfil 2).

Comparando las tablas 4,5 y 6 , se establece lo siguiente: para los sismos registrados en roca, se observa que la duración de las frecuencias dominantes oscila en un rango de 0.50 segundos, excepto para el sismo del 10 de abril de 2006 (10/04/06) en la componente vertical registra un tiempo de 3.70 segundos. Para las señales correspondientes al suelo aluvial (perfil 1), determinamos que estas aumentan los tiempos de duración de las frecuencias dominantes, en

Tabla 2. Frecuencias dominantes (en Hertz) según el tipo de fuente.

\begin{tabular}{|c|c|c|c|c|}
\hline \multirow{2}{*}{ Fuente } & \multicolumn{2}{|c|}{ Componente EW } & \multicolumn{2}{c|}{ Componente Vertical } \\
\cline { 2 - 5 } & Roca & Suelo & Roca & Suelo \\
\hline Nido de Bucaramanga & $3.8-6.2$ & 1.0 & $4.0-6.8$ & $0.8-1.0$ \\
\hline D. E. $<200 \mathrm{Km}$ & 4.0 & $1.2-1.6$ & $5.0-5.5$ & $0.8-0.9$ \\
\hline D. E. $>200 \mathrm{Km}$ & $2.5-3.0$ & & $2.8-3.0$ & \\
\hline
\end{tabular}

Fuente: Autores.

Tabla 3. Frecuencias dominantes (en Hertz) según el perfil de suelo analizado.

\begin{tabular}{|c|c|c|}
\hline Componente & Este - Oeste & Vertical \\
\hline Suelo & & \\
\hline Perfil 1 & $3.0-6.0$ & $3.0-7.0$ \\
\hline Perfil 2 & $3.0-4.0$ & $3.0-5.0$ \\
\hline
\end{tabular}

Fuente: Autores. 


\section{ANÁLISIS Y TRATAMIENTO DE SEÑALES DE FUENTES SISMOGÉNICAS DE CAMPO CERCANO A SAN JOSÉ DE CÚCUTA, COLOMBIA}

cambio el perfil de suelo arcilloso (perfil 2 ), mantiene aproximadamente la misma duración en el tiempo de las frecuencias respecto a la señal registrada en roca. Para la señal captada en suelo (tabla 7), se observa que en promedio las duraciones en el tiempo de las frecuencias dominantes se acercan a los 0.4 segundos.

Tabla 4. Duración de frecuencias según la WT - Roca.

\begin{tabular}{|c|c|c|c|c|}
\hline Señal en Roca & \multicolumn{2}{|c|}{ Componente EW } & \multicolumn{2}{c|}{ Componente Vertical } \\
\hline Sismo & Frecuencia & Duración (seg) & Frecuencia & Duración (seg) \\
\hline $\mathbf{0 1 / 0 1 / 1 9 9 7}$ & Bajas & 0.46 & Bajas & 0.50 \\
\hline $\mathbf{2 1 / 0 2 / 1 9 9 7}$ & Bajas & 0.49 & Bajas & 0.50 \\
\hline $\mathbf{1 0 / 0 4 / 2 0 0 6}$ & Bajas & 0.54 & Bajas & 3.70 \\
\hline
\end{tabular}

Fuente: Autores.

Tabla 5. Duración de frecuencias según la WT - Perfil 1.

\begin{tabular}{|c|c|c|c|c|}
\hline Perfil 1 & \multicolumn{2}{|c|}{ Componente EW } & \multicolumn{2}{c|}{ Componente Vertical } \\
\hline Sismo & Frecuencia & Duración (seg) & Frecuencia & Duración (seg) \\
\hline $\mathbf{0 1 / 0 1 / 1 9 9 7}$ & Bajas & 0.49 & Bajas & 1.68 \\
\hline $\mathbf{2 1 / 0 2 / 1 9 9 7}$ & Bajas & 0.97 & Bajas & 0.60 \\
\hline $\mathbf{1 0 / 0 4 / 2 0 0 6}$ & Bajas & 2.10 & Bajas & 4.00 \\
\hline
\end{tabular}

Fuente: Autores.

Tabla 6. Duración de frecuencias según la WT - Perfil 2.

\begin{tabular}{|c|c|c|c|c|}
\hline Perfil 2 & \multicolumn{2}{|c|}{ Componente EW } & \multicolumn{2}{c|}{ Componente Vertical } \\
\hline Sismo & Frecuencia & Duración (seg) & Frecuencia & Duración (seg) \\
\hline $\mathbf{0 1 / 0 1 / 1 9 9 7}$ & Bajas & 0.43 & Bajas & 0.52 \\
\hline $\mathbf{2 1 / 0 2 / 1 9 9 7}$ & Bajas & 0.48 & Bajas & 0.42 \\
\hline $\mathbf{1 0 / 0 4 / 2 0 0 6}$ & Bajas & 0.53 & Bajas & 3.70 \\
\hline
\end{tabular}

Fuente: Autores.

Tabla 7. Duración de frecuencias según la WT - Suelo.

\begin{tabular}{|c|c|c|c|c|}
\hline Señal en Suelo & \multicolumn{2}{|c|}{ Componente EW } & \multicolumn{2}{c|}{ Componente Vertical } \\
\hline Sismo & Frecuencia & Duración (seg) & Frecuencia & Duración (seg) \\
\hline $17 / 07 / 1996$ & Media & 0.75 & Bajas & 0.43 \\
\hline $31 / 08 / 1996$ & Bajas & 0.41 & Bajas & 0.40 \\
\hline
\end{tabular}

Fuente: Autores. 


\section{ANÁLISIS Y TRATAMIENTO DE SEÑALES DE FUENTES SISMOGÉNICAS DE CAMPO CERCANO A SAN JOSÉ DE} CÚCUTA, COLOMBIA

4.3. Resultados para el cálculo de espectros de respuesta

En las siguientes tablas, se hace un resumen de los valores correspondientes a los periodos dominantes de las señales sísmicas en las componentes EW y Vertical, así como la determinación de la aceleración máxima y el periodo asociado a este valor.

De la tabla 8, se observa que los periodos dominantes se conservan en un rango aproximado entre 0.1 y 0.3 segundos para los registros en roca y suelo aluvial, en cambio, para el suelo arcilloso, se observa que este presenta un periodo de 0.25 segundos. Los periodos correspondientes a las aceleraciones máximas se concentran alrededor de 0.25 segundos, para las señales en roca y los perfiles de suelo analizados. Para el registro captado en suelo, se aprecia un rango de periodos dominantes entre 0.05 y 0.38 segundos, presentando la aceleración máxima en un periodo de 0.25 segundos.

Para la componente vertical, que se presenta en la tabla 9, observamos que para el registro en roca y para la señal analizada en suelo aluvial (perfil 1), los periodos dominantes son similares, en cambio el suelo arcilloso (perfil 2) exhibe dos periodos dominantes bien definidos, correspondientes a 0.10 y 0.28 segundos. Lo que quiere decir que la señal no se ve afectada en sus periodos dominantes al atravesar el suelo aluvial, se percibe una amplificación de la aceleración que se presenta cuando la señal llega a la superficie. Para el registro en suelo se observa un rango de periodos dominantes entre 0.03 y 0.25 segundos, además el periodo asociado a la aceleración máxima se encuentra en 0.03 segundos.

Tabla 8. Periodos dominantes y aceleración espectral máxima Componente EW.

\begin{tabular}{|c|c|c|c|c|}
\hline \multirow{2}{*}{$\begin{array}{c}\text { Espectro de } \\
\text { Respuesta }\end{array}$} & \multicolumn{4}{|c|}{ Componente EW } \\
\cline { 2 - 5 } & Roca & Suelo & Perfil 1 & Perfil 2 \\
\hline Periodos Dominantes (seg) & $0.1-0.3$ & $0.05-0.38$ & $0.15-0.33$ & 0.25 \\
\hline A max (g) & 0.048 & 0.036 & 0.100 & 0.100 \\
\hline Periodo máximo (seg) & 0.25 & 0.25 & 0.25 & $0.25-0.30$ \\
\hline
\end{tabular}

Fuente: Autores.

Tabla 9. Periodos dominantes y aceleración espectral máxima Componente Vertical.

\begin{tabular}{|c|c|c|c|c|}
\hline \multirow{2}{*}{$\begin{array}{c}\text { Espectro de } \\
\text { Respuesta }\end{array}$} & \multicolumn{4}{|c|}{ Componente Vertical } \\
\cline { 2 - 5 } & Roca & Suelo & Perfil 1 & Perfil 2 \\
\hline Periodos Dominantes (seg) & $0.05-0.3$ & $0.03-0.25$ & $0.05-0.3$ & 0.10 y 0.28 \\
\hline A max (g) & 0.026 & 0.051 & 0.059 & 0.047 \\
\hline Periodo máximo (seg) & 0.25 & 0.03 & 0.15 & 0.25 \\
\hline
\end{tabular}

Fuente: Autores. 


\section{ANÁLISIS Y TRATAMIENTO DE SEÑALES DE FUENTES SISMOGÉNICAS DE CAMPO CERCANO A SAN JOSÉ DE CÚCUTA, COLOMBIA}

\section{CONCLUSIONES}

Las señales sísmicas correspondientes al Nido de Bucaramanga registradas en roca, exhiben un rango de frecuencias entre 3.8 y $6.8 \mathrm{~Hz}$ para ambas componentes de registro. Los registros en suelo que corresponden a esta fuente sismogénica muestran valores cercanos a $1.0 \mathrm{~Hz}$ para las dos componentes de registro.

Para los sismos con distancias epicentrales menores a $200 \mathrm{Km}$ registrados en roca, muestran una frecuencia de $4.0 \mathrm{~Hz}$ para la componente Este - Oeste y un rango de frecuencias entre 5.0 y $5.5 \mathrm{~Hz}$ para la componente Vertical. Las señales sísmicas captadas en superficie presentan bajos valores de frecuencia, aproximadamente entre 0.8 y $1.6 \mathrm{~Hz}$ para ambas componentes de registro.

Las señales sísmicas que pertenecen a distancias epicentrales mayores a $200 \mathrm{Km}$, exhiben valores de frecuencias entre 2.5 y $3.0 \mathrm{~Hz}$, para las dos componentes de registro de las señales sísmicas en roca.

Las frecuencias encontradas para el perfil de suelo aluvial (perfil 1), presenta un rango de frecuencias entre 3.0 y $7.0 \mathrm{~Hz}$, el cual, muestra una alta similitud con las frecuencias determinadas para los registros en roca, percibiendo que este perfil de suelo no modifica las componentes frecuenciales de la señal cuando atraviesa una capa de suelo de estas características. El perfil de suelo arcilloso (perfil 2) exhibe valores entre 3.0 y $4.0 \mathrm{~Hz}$ para la componente Este - Oeste; entre 3.0 y $5.0 \mathrm{~Hz}$ para la componente Vertical, por lo cual, podemos inferir que, la señal, al propagarse por este perfil de suelo es modificada en su contenido frecuencial, agrupando todas las frecuencias en un rango determinado entre 3.0 y 5.0 $\mathrm{Hz}$ aproximadamente.

En el análisis para la transformada wavelet, se observa la tendencia a bajas frecuencias por parte de las componentes frecuenciales dominantes de la señal sísmica, además, de la corta duración de estas frecuencias en el tiempo, las cuales, no superan un segundo en la mayoría de las señales analizadas. En todos los casos, la fase intensa del sismo coincide con la duración de las componentes frecuenciales dominantes de la señal sísmica, estableciendo que una larga duración de las frecuencias dominantes en el tiempo, puede producir una mayor afectación a las estructuras, al tener que oscilar en un mayor rango de tiempo.

Comparando la duración en el tiempo de las frecuencias dominantes entre los perfiles de suelo y los registros en roca, se establece que el perfil de suelo aluvial (perfil 1) modifica la duración en el tiempo hacia valores mayores con respecto a la señal en roca, y el perfil de suelo arcilloso (perfil 2) presenta un fenómeno contrario, ya que este mantiene los mismos valores de duración en el tiempo para las frecuencias dominantes.

Los periodos dominantes que se extraen de los espectros de respuesta para la componente Este - Oeste presentan valores aproximados entre 0.1 y 0.3 segundos para las señales en roca y en el suelo aluvial, en cambio para el suelo arcilloso aparece un único valor correspondiente a 0.25 segundos; para el registro en suelo se establece un rango de periodos dominantes entre 0.05 y 0.38 segundos. En la componente vertical del sismo se encuentran valores similares entre el registro en roca y el perfil de suelo aluvial, presentando periodos entre $0.05 \mathrm{y}$ 3.0 segundos. Los periodos dominantes para el suelo arcilloso exhibe dos periodos bien definidos que corresponden a 0.10 y 0.28 segundos. El registro en suelo presenta un rango de periodos dominantes entre 0.03 y 0.25 segundos. Estableciendo que la señal no se ve afectada en sus periodos dominantes al atravesar el perfil de suelo aluvial, en cambio el suelo arcilloso presenta una tendencia a agrupar los valores de periodos dominantes a un rango determinado, tales como $0.10,0.25$ y 0.28 segundos. 


\section{ANÁLISIS Y TRATAMIENTO DE SEÑALES DE FUENTES SISMOGÉNICAS DE CAMPO CERCANO A SAN JOSÉ DE} CÚCUTA, COLOMBIA

Los valores de periodos dominantes correspondientes a la señal registrada en suelo (estación ubicada sobre suelo aluvial) con el perfil de suelo aluvial analizado, muestran similitud para las dos componentes de registro, por lo cual, la modelación teórica realizada puede estar acercándose a la realidad.

Las frecuencias dominantes calculadas en el espectro de Fourier, al determinarse el periodo, coincide con el rango de periodos dominantes del espectro de respuesta. Además, puede observarse que la aceleración espectral máxima aumenta el doble con respecto a la señal registrada en roca, estableciendo que estos suelos analizados para la ciudad de Cúcuta son amplificadores de la señal sísmica.

En el análisis para sismos de movimiento fuerte, se observa que los suelos aluviales (perfil 1), amplifican la señal para periodos altos, mientras que los suelos que corresponden a suelos arcillosos (perfil 2), amplifican para bajos periodos pero atenúan la aceleración de la señal para altos periodos, es decir, para bajas frecuencias.

\section{BIBLIOGRAFÍA}

[1] Clough, Ray W., Penzien, Joseph (2003). “Dynamics of Structures". 3a edición, Computers and Structures Inc., Berkeley, California, USA.

[2] Dowrick, D. J. (1992). "Diseño de Estructuras Resistentes a Sismos". 2a edición, Editorial Limusa S.A., México, D. F.

[3] Gallego Silva, Mauricio (2003). "Terremotos Causas, Conceptos, Consecuencias y Prevención". 1a edición. Universidad de los Andes, Bogotá D.C., Colombia. ISBN 958-695- 071-9.

[4] Sarria Molina, Alberto (2002). "Fundamentos de Ingeniería Sísmica” (Capítulo 7). Universidad de los Andes. Bogotá, Colombia. ISBN 958- 695- 067-
0 http://ingsismica.uniandes.edu.co (consultado en Abril de 2006).

[5] Ambraseys, N., Douglas, J. (2000). "Reappraisal of the effect of vertical ground motions on response". Engineering Seismology and Earthquake Engineering, Imperial College of Science, Technology and Medicine, Civil Engineering Department, London.

[6] Herráiz Sarachaga, Miguel (1997). “Conceptos Básicos de Sismología para Ingenieros". 1a edición, Universidad Nacional de Ingeniería - CISMID, Lima, Perú.

http://www.cismid.uni.edu.pe/descargas/ publicaciones/publi_3.zip (consultado en Junio de 2007).

[7] "Normas Colombianas de Diseño y Construcción Sismo Resistente (NSR 98)", Ley 400 de 1997. República de Colombia.

[8] Asociación de Ingeniería Sísmica, Comité AIS 300 (1996). "Estudio General de Amenaza Sísmica de Colombia". Bogotá D.C., Colombia.

[9] Alcaldía Mayor de Bogotá D.C., Centro de Estudios sobre Desastres y Riesgos - CEDERI (2002). "Procesamiento e Interpretación de la Información Sísmica de Bogotá D.C.". Universidad de los Andes, Bogotá D.C., Colombia.

[10] Bath, Markus (1974). "Spectral Analysis in Geophysics". 1a edición, Seismological Institute, University of Upssala, Sweden. ISBN 0-444-41799 -0 .

[11] Instituto Colombiano de Geología y Minería - Ingeominas, Red Nacional de Acelerógrafos de Colombia - RNAC. (1997). "Boletín de Movimiento Fuerte". Bogotá D.C., Colombia. http://www. ingeominas.gov.co/index.php?option=com wrapper\&Itemid=313 (consultado en Agosto de 2006). 


\section{ANÁLISIS Y TRATAMIENTO DE SEÑALES DE FUENTES SISMOGÉNICAS DE CAMPO CERCANO A SAN JOSÉ DE CÚCUTA, COLOMBIA}

[12] Kuo, Sen M., Lee, Bob H. (2001). "Real - Time Digital Signal Processing". Jhon Wiley and Sons, England. ISBN 0 - 470 - 84534 - 1 .

[13] Smith, Steven W. (1999) "The Scientist and Engineer's Guide to Digital Signal Processing". 2a edición, California Technical Publishing, San Diego, California, USA. ISBN 0-9660176-6-8. www. dspguide.com (consultado en Julio de 2006).

[14] "SeismoSignal - A computer program for signal processing of strong-motion data" [online]. Available [2004] from URL: http://www.seismosoft.com (consultado en Agosto de 2006).

[15] Instituto Colombiano de Geología y Minería (INGEOMINAS), Empresa Industrial y Comercial de Servicios Públicos de Cúcuta (E. I. S.). (2004). "Estudio de Estabilidad de la Ladera sobre la que se encuentra la Estación de Bombeo y Tanques de la Nidia Cúcuta". San José de Cúcuta, Colombia.

[16] Rodríguez, Guillermo., Santander, Karen (2006). “Caracterización del Macizo Arcilloso Localizado en el Costado Sur Occidental del Sector Doña Nidia de la Ciudad de Cúcuta". Tesis de Pregrado. Plan de estudios de Ingeniería de Minas, Universidad Francisco de Paula Santander, San José de Cúcuta, Colombia.

[17] Sarmiento, M. et al. (2004). "Base Metodológica Preliminar de la Microzonificación Sísmica de Cúcuta". Tesis de Pregrado. Plan de Estudios de Ingeniería Civil, Universidad Francisco de Paula Santander, San José de Cúcuta, Colombia.

[18] Florez G, C.H, Pedroza R, A.O (2006). “Desarrollo de estudios preliminares para la microzonificación sísmica de San Jose de Cucuta, Colombia". C.D. ISBN 958-33-9649-4. Simposio Latinoamericano y del Caribe en Geofisica. Bogota D.C., Colombia.

[19] Das, Braja M. (1993). "Principles of Soil Dynamics". Editorial Brooks/Cole, USA.
[20] Bardet, J. P., et al (2000). "A Computer Program for Equivalent-linear Earthquake site Response Analyses of Layered Soil Deposits - EERA". Department of Civil Engineering, University of Southern California.

http://gees.usc.edu/GEES/Software/EERA2000/

Default.htm (consultado en Noviembre de 2005).

[21] Ordaz, M., Montoya, C. (2002). "Programa DEGTRA A4 versión 4.06". Instituto de Ingeniería, Universidad Nacional Autónoma de México, México.

[22] Charney, Finley A, et al. (2006). "Programa NONLIN versión 6.01". Advanced Structural Concepts, Federal Emergency Management Agency (FEMA), Denver, Colorado, USA. http://training.fema. gov/EMIWeb/nonlin.asp (consultado en Agosto de 2006).

[23] EduPro Civil Systems (1999). "Programa EduShake". Redmond, Washington, USA. www. proshake.com (consultado en Agosto de 2006).

[24] Haykin, Simon, Van Veen, Barry (2006). "Señales y Sistemas". Editorial Limusa y Jhon Wiley and Sons, México. ISBN 968-18-5914- 6 .

[25] Irarrázaval, Pablo (1999). "Análisis de Señales". Editorial Mc Graw Hill, Chile.

[26] Penrose, Roger (2007). "El Camino a la Realidad". Editorial Debate, México.

[27] Proakis, Jhon G., Ingle, Vinay K. (1997). "Digital Signal Processing Using Matlab v.4". International Thomson Publishing, USA. ISBN $0-534-93805$ -1 .

[28] Rodríguez, M. (2005). “Caracterización de la Respuesta Sísmica de los Suelos". Aplicación a la ciudad de Barcelona, Ph.D. Thesis. Universidad Politécnica de Cataluña, Barcelona, España.

[29] Castro Fernández, Rosa María de. (2002). 
"Análisis de la Teoría de Ondículas Orientada a las Aplicaciones en Ingeniería Eléctrica: Fundamentos". Ph.D. Thesis, Universidad Politécnica de Madrid, Madrid, España.

[30] Cuesta Frau, David (2001). "Estudio de Métodos para Procesamiento y Agrupación de Señales Electrocardiográficas". Ph.D. Thesis, Universidad Politécnica de Valencia, Valencia, España.

[31] Mallat, Stéphane (1999). "A Wavelet Tour of Signal Processing". 2a edición, Academic Press, San Diego, California, USA. ISBN 0-12-466606-X.

[32] Mertins, Alfred (1999). "Signal Analysis: Wavelets, Filter Banks, Time - Frequency Transforms and applications". Jhon Wiley and Sons, England. ISBN 0 $-470-84183-4$.

[33] Misiti, Michel et al (1997). "Wavelet toolbox for use with Matlab user's guide". The MathWorks. USA. www.mathworks.com (consultado en Mayo de 2006).

[34] Benito, B., Cabañas, L (2003). “Caracterización del Movimiento del Suelo en Ingeniería Sísmica". E. U. I. T. de Topografía, Universidad Politécnica de Madrid, Madrid, España.

[35] Crisafulli, F., Villafañe, E. (2002). "Espectros de Respuesta y de Diseño". Facultad de Ingeniería, Universidad Nacional de Cuyo, Mendoza, Argentina.

Fecha de recibido: Agosto 15 de 2007

Fecha de aceptación: Diciembre 14 de 2007 


\section{ANÁLISIS Y TRATAMIENTO DE SEÑALES DE FUENTES SISMOGÉNICAS DE CAMPO CERCANO A SAN JOSÉ DE CÚCUTA, COLOMBIA}

\section{ANEXO 1. SEÑALES SÍSMICAS REGISTRADAS EN LA CIUDAD DE SAN JOSÉ DE CÚCUTA}
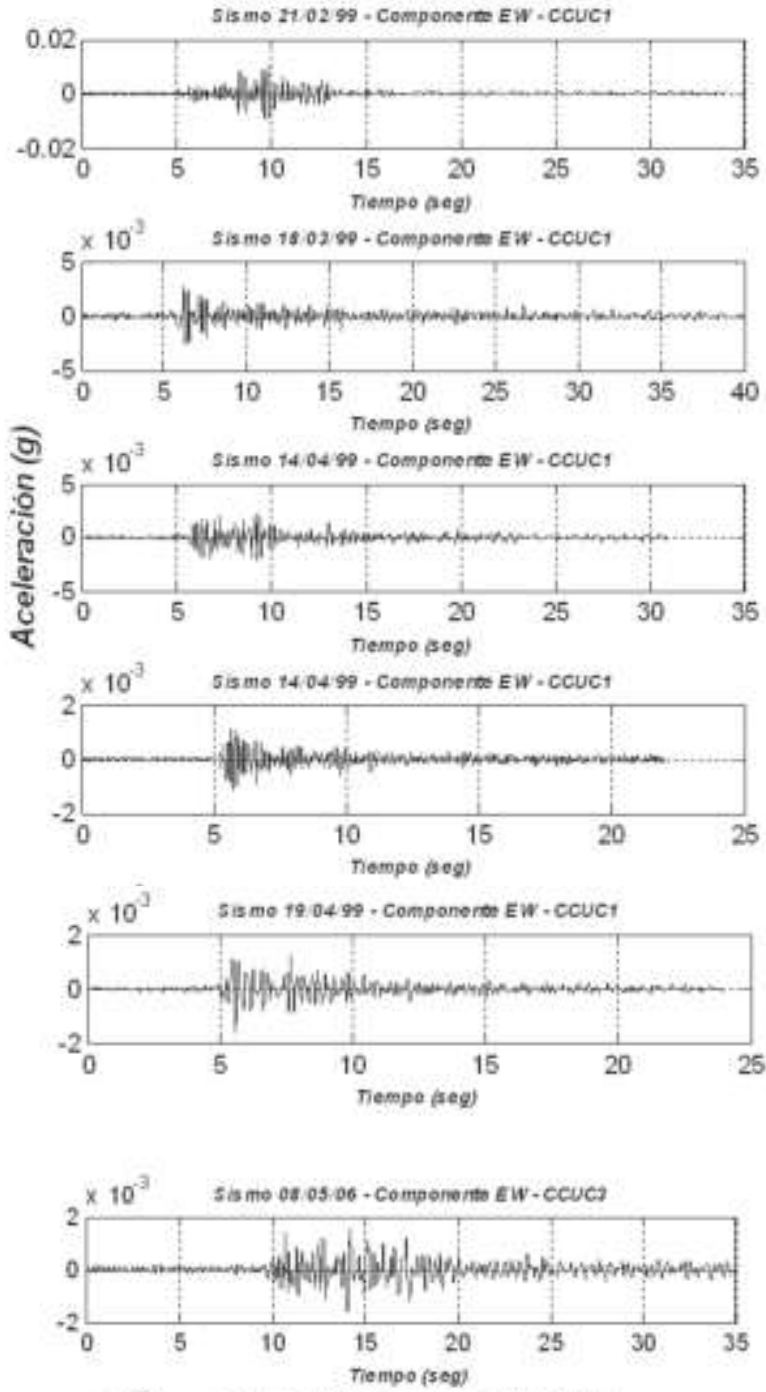

$510^{-3}$ sisme ot 05.06-Componerne EW- cevea
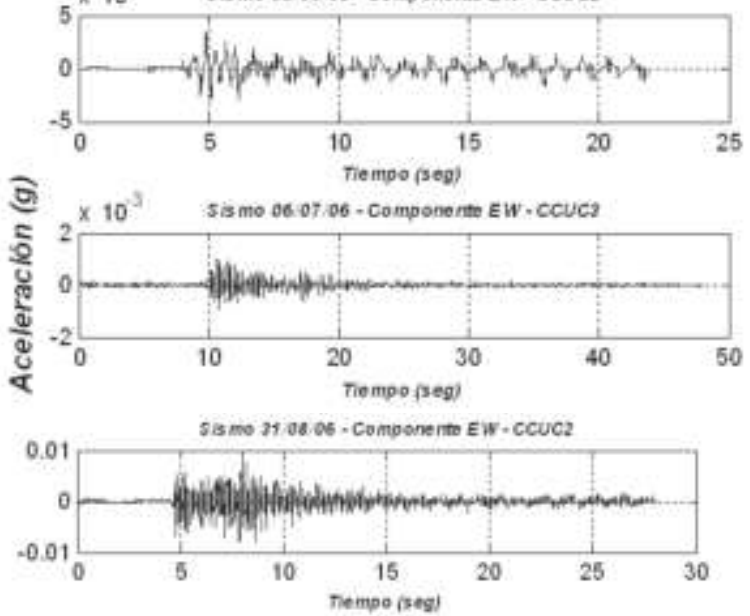
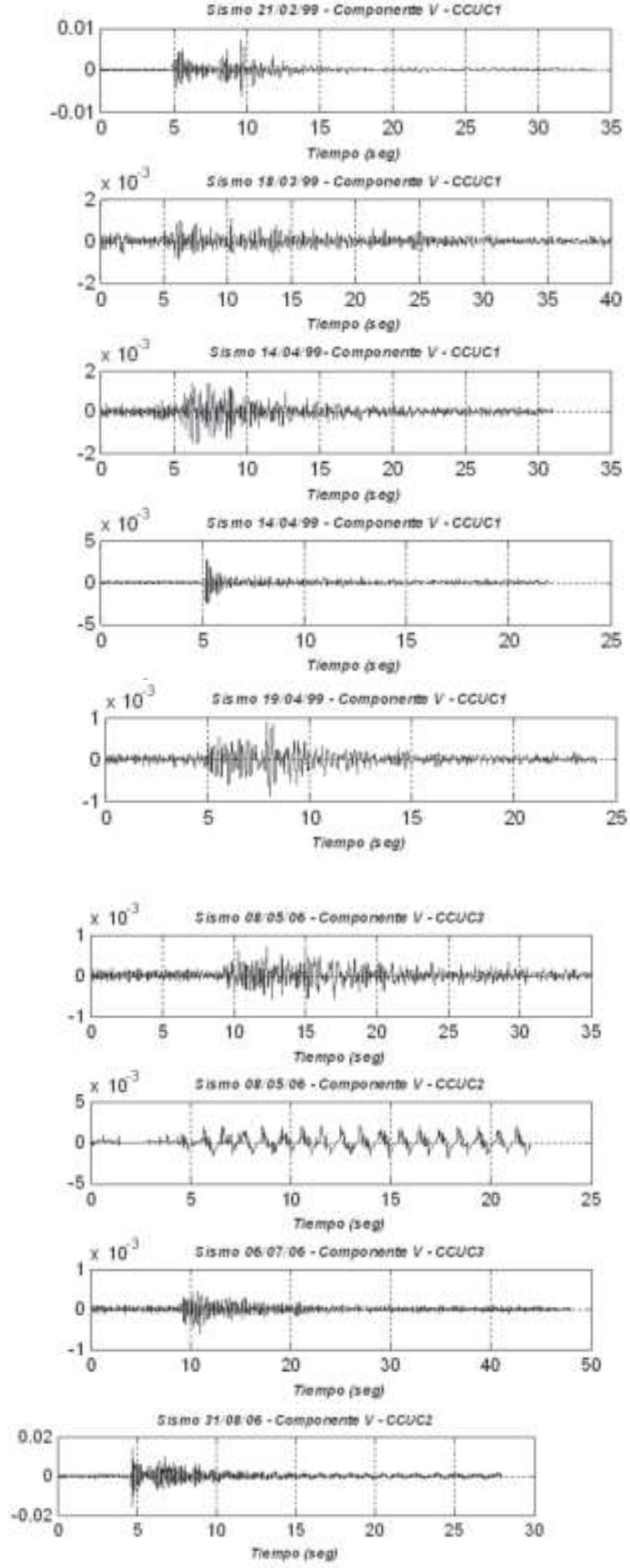

DE CUUCUTA, COLOMBIA

ANEXO 2. CONTENIDO FRECUENCIAL DE LAS SEÑALES SÍSMICAS

Nido de Bucaramanga
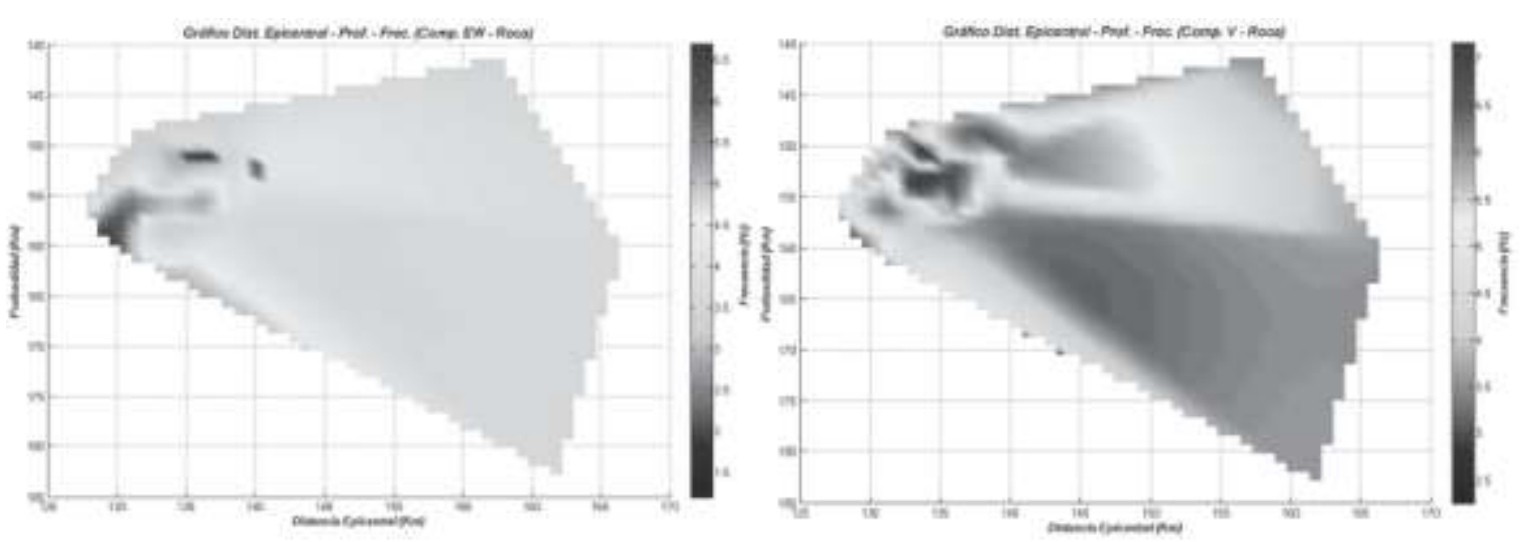

Sismos con distancias epicentrales menores a $200 \mathrm{Km}$
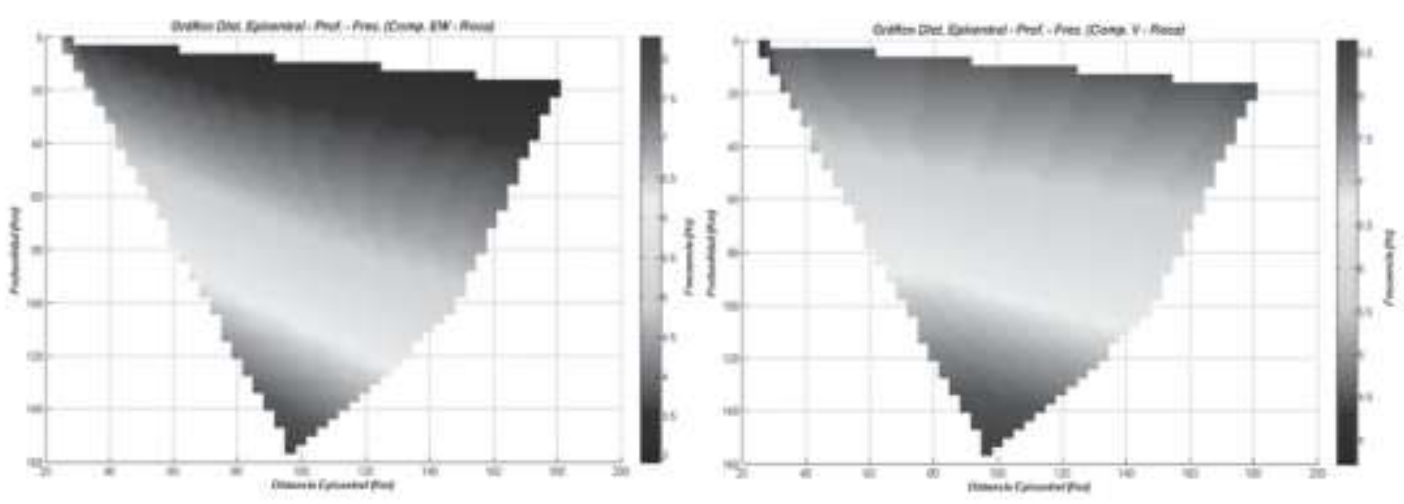

Sismos con distancias epicentrales mayores a $200 \mathrm{Km}$
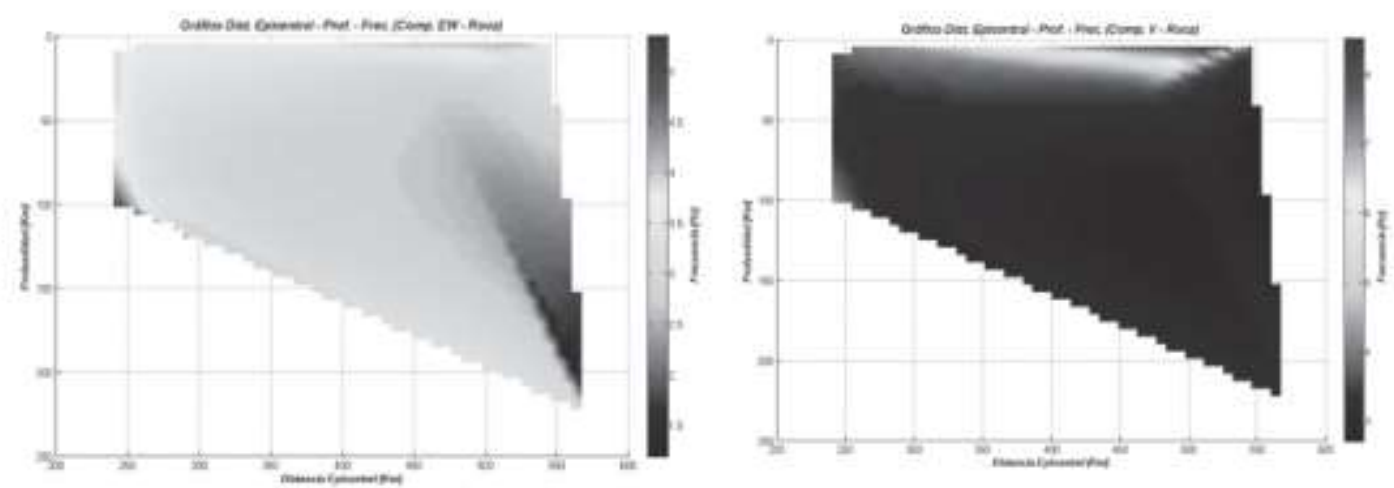


\section{ANÁLISIS Y TRATAMIENTO DE SEÑALES DE FUENTES SISMOGÉNICAS DE CAMPO CERCANO A SAN JOSÉ DE CÚCUTA, COLOMBIA}

\section{ANEXO 3. ESPECTROS DE FOURIER}

Espectros de Fourier
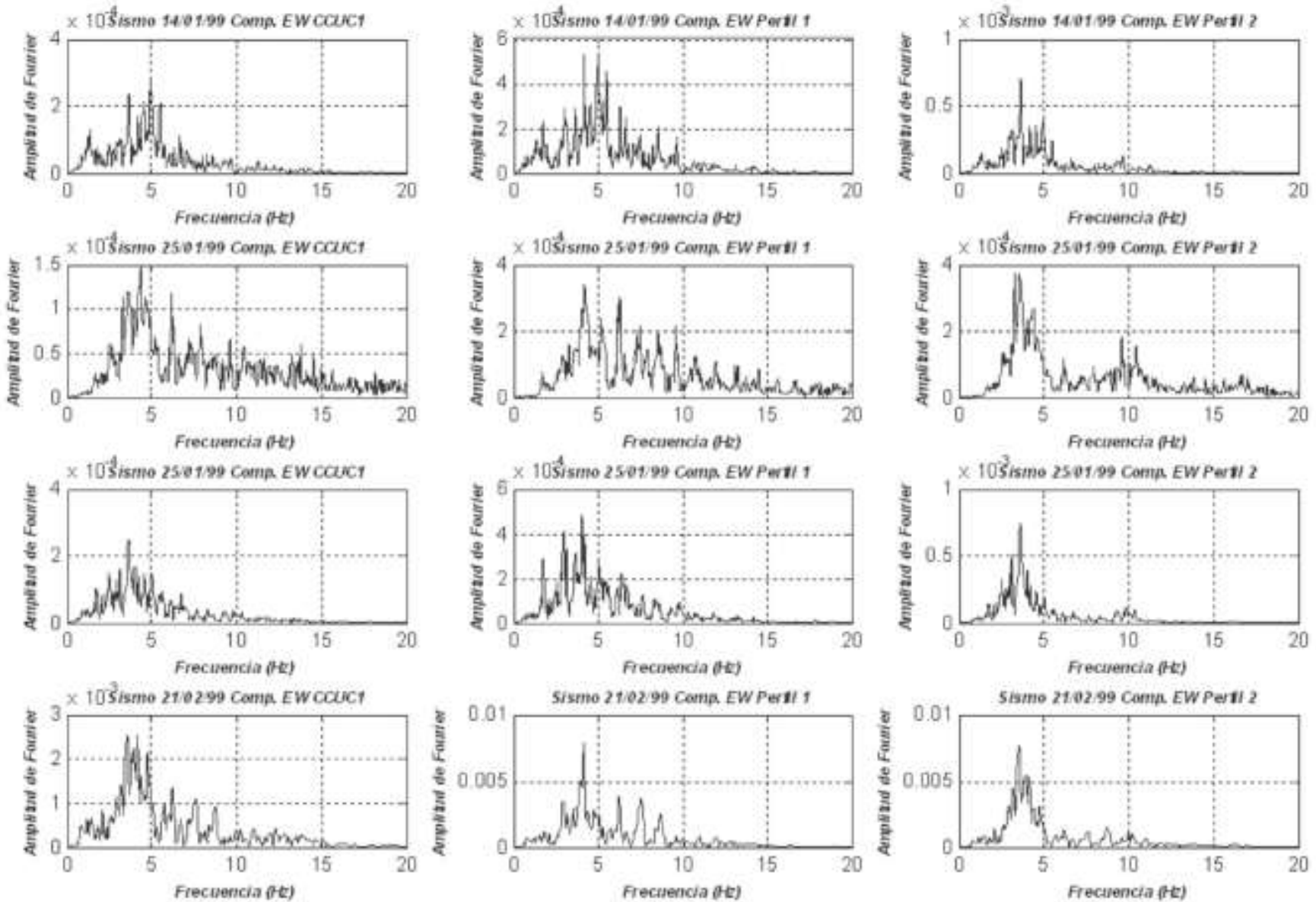

Conjunto de Espectros de Fourier
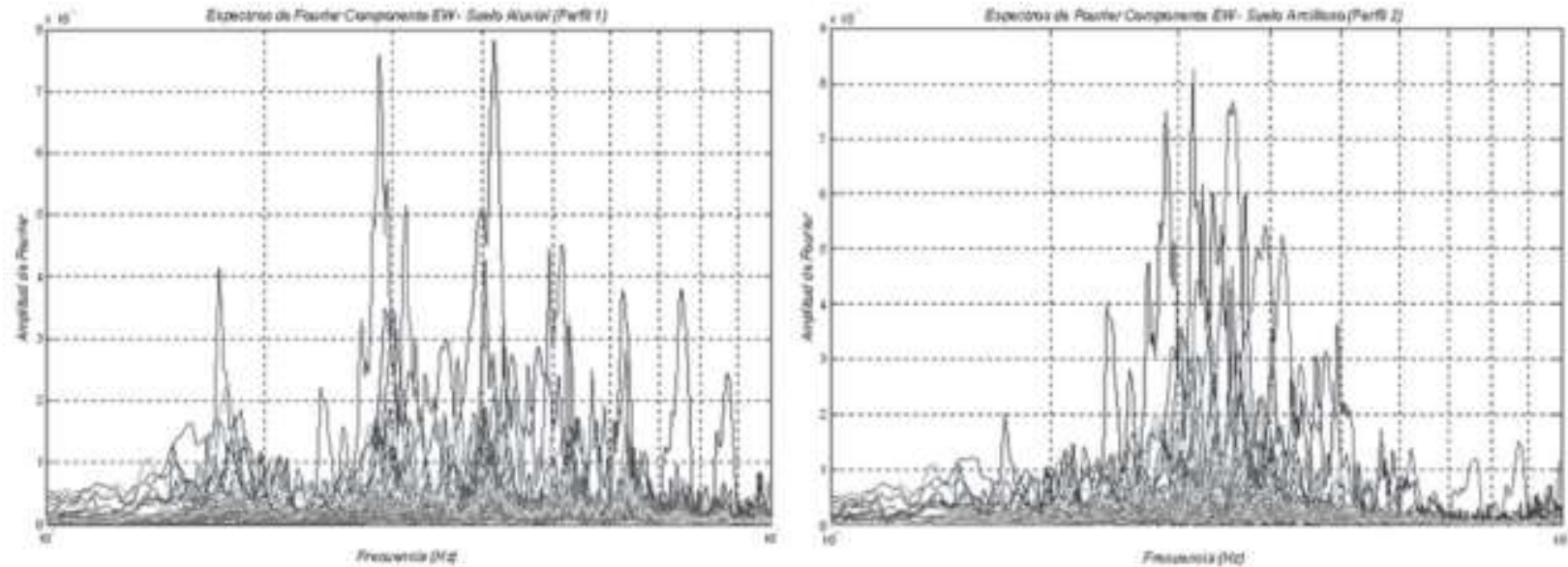
ANÁLISIS Y TRATAMIENTO DE SEÑALES DE FUENTES SISMOGÉNICAS DE CAMPO CERCANO A SAN JOSÉ DE CÚCUTA, COLOMBIA

\section{ANEXO 4. ESPECTROS PARA LA TRANSFORMADA WAVELET}

Espectro de la transformada wavelet para el sismo del 21 de febrero de 1999 Componente EW - Roca

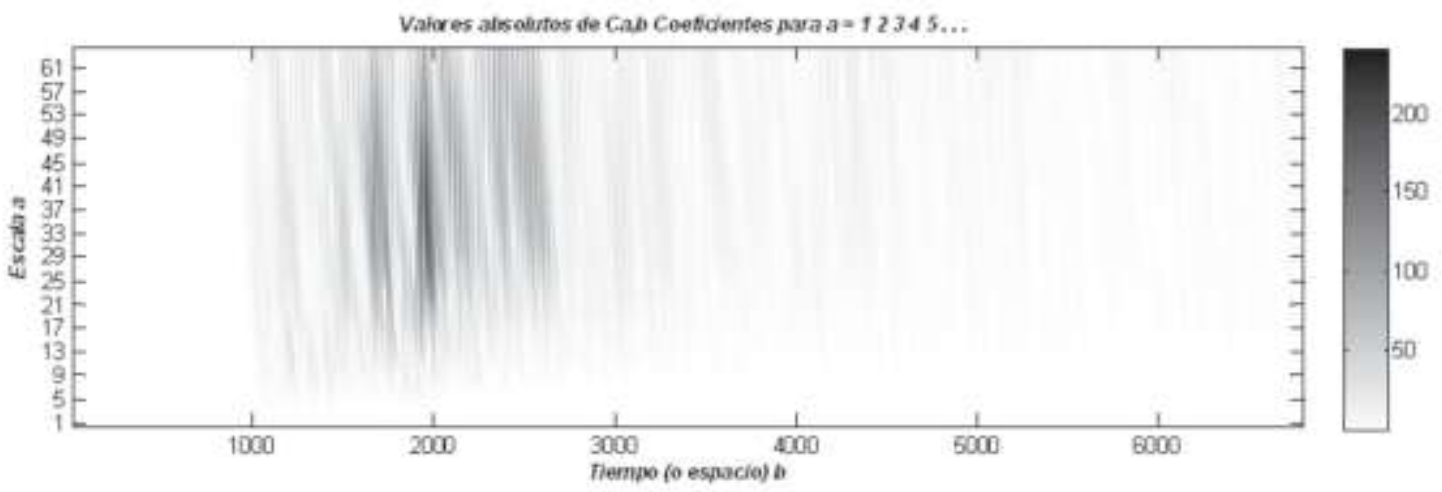

Espectro de la transformada wavelet para el sismo del 21 de febrero de 1999 Componente EW - Suelo Aluvial (Perfil 1)

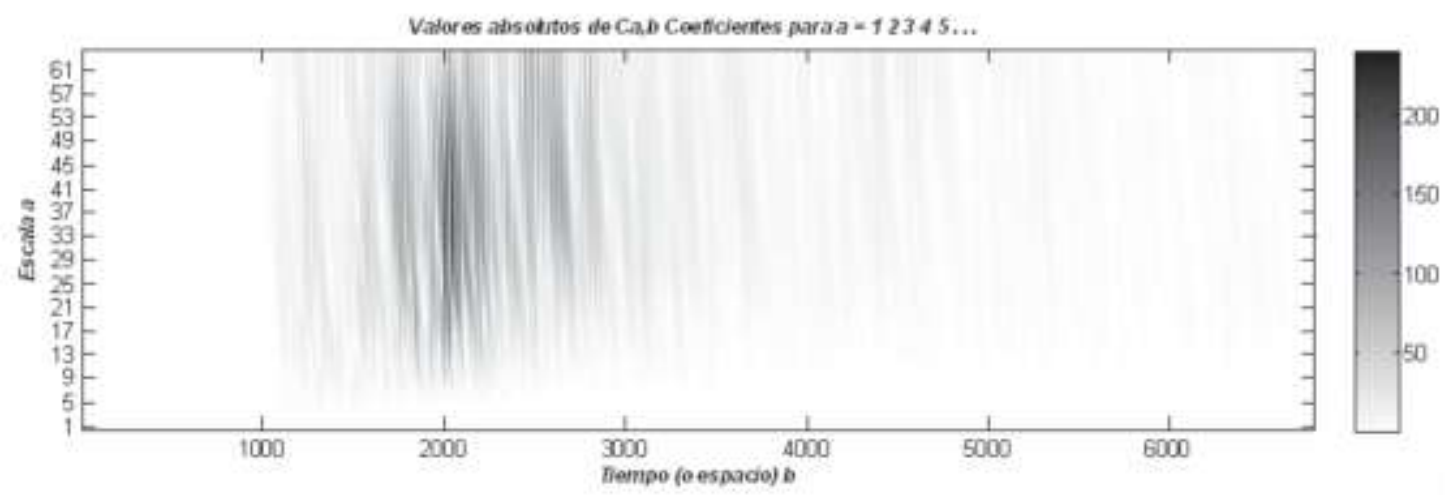

Espectro de la transformada wavelet para el sismo del 21 de febrero de 1999 Componente EW - Suelo Arcilloso (Perfil 2)

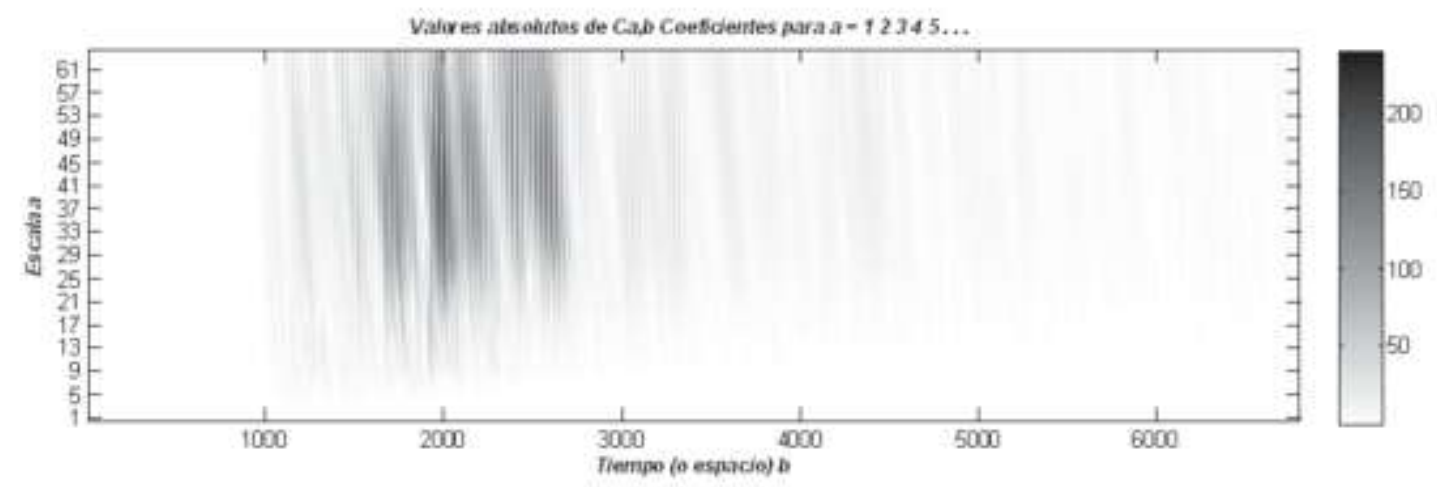


ANÁLISIS Y TRATAMIENTO DE SEÑALES DE FUENTES SISMOGÉNICAS DE CAMPO CERCANO A SAN JOSÉ DE CÚCUTA, COLOMBIA

\section{ANEXO 5. ESPECTROS DE RESPUESTA}
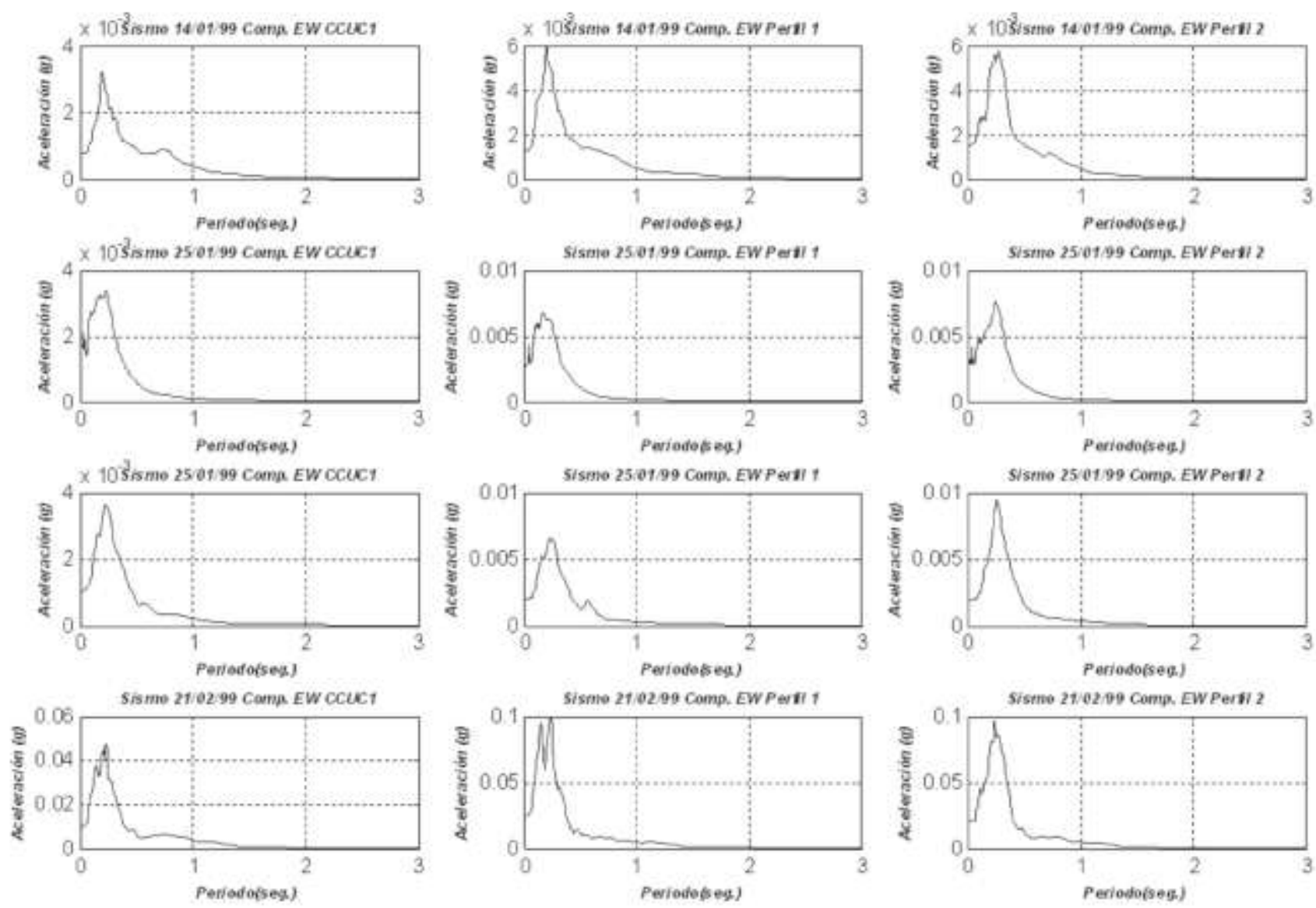

Conjunto de Espectros de Respuesta
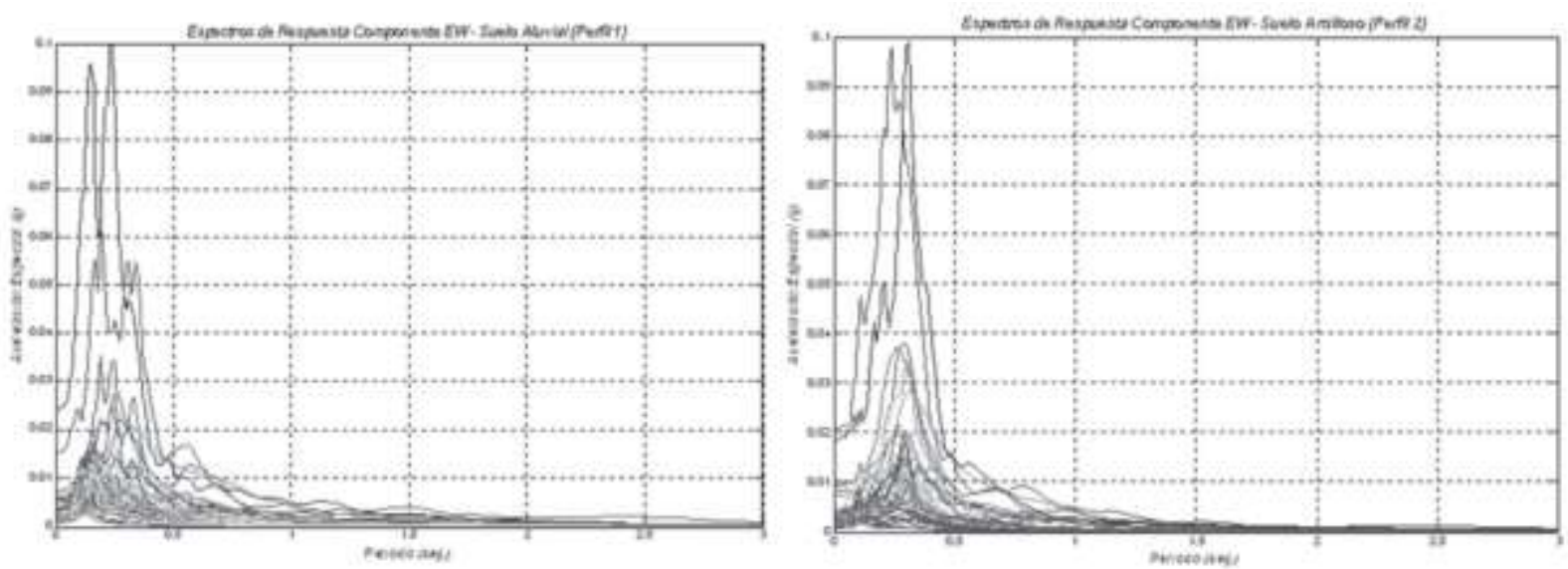
ANÁLISIS Y TRATAMIENTO DE SEÑALES DE FUENTES SISMOGÉNICAS DE CAMPO CERCANO A SAN JOSÉ DE CÚCUTA, COLOMBIA

ANEXO 6. ESPECTROS DE RESPUESTA PARA SISMOS DE MOVIMIENTO FUERTE
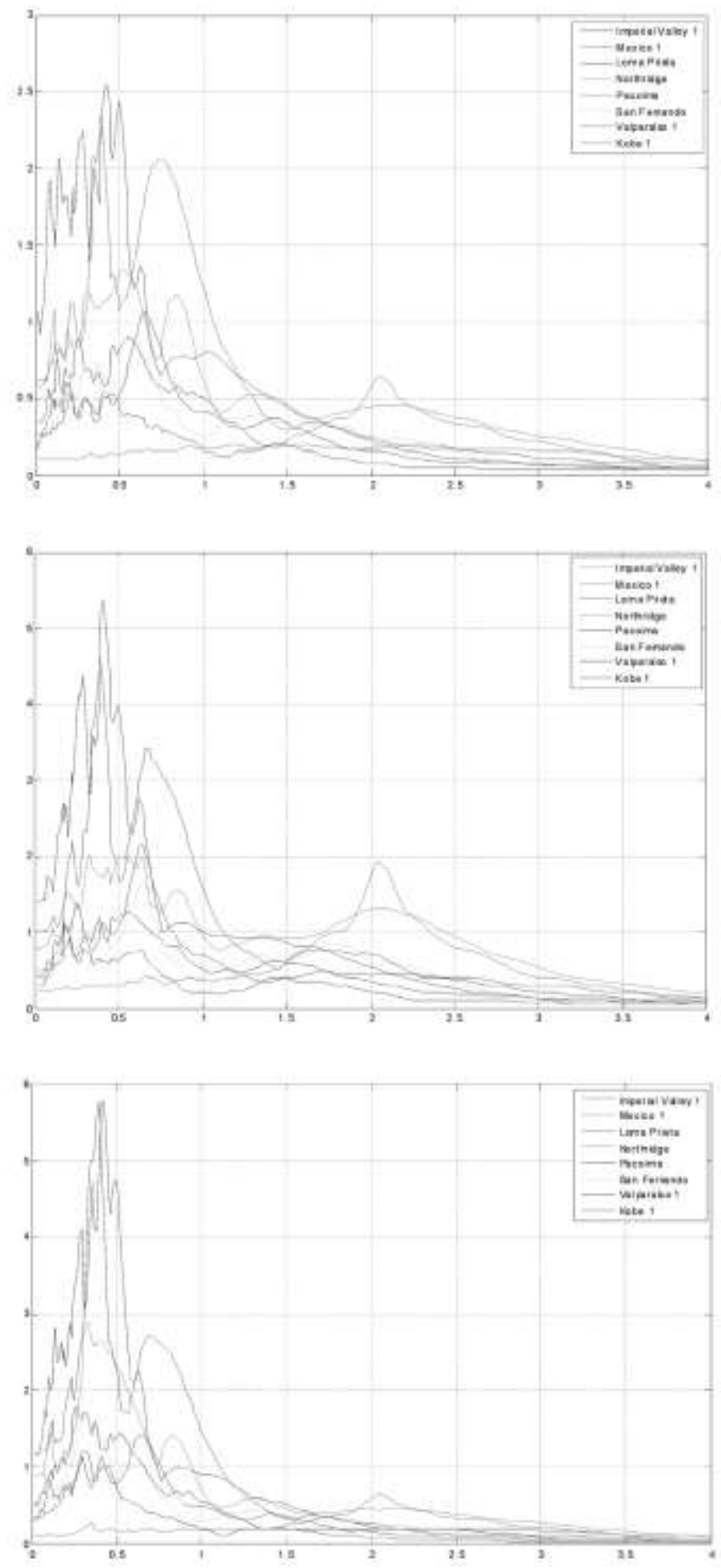